\title{
Impacto del tabaquismo y la EPOC sobre el funcionamiento cerebral
}

\author{
Valeri Noé-Díaz,* ${ }^{*}$ Leonor García-Gómez, ${ }^{*}$ Raúl H Sansores, * Alejandra Ramírez-Venegas*
}

*Departamento de Investigación en Tabaquismo y EPOC, Instituto Nacional de Enfermedades Respiratorias Ismael Cosío Villegas; Universidad Nacional Autónoma de México.

Trabajo recibido: 26-VI-2014; aceptado: 27-VIII-2014

\begin{abstract}
RESUMEN. Existen diversos padecimientos y factores que pueden tener un impacto sobre el funcionamiento cerebral debido a los cambios fisiológicos en estructuras cerebrales. En la práctica clínica, las enfermedades respiratorias, incluyendo el tabaquismo y la enfermedad pulmonar obstructiva crónica (EPOC) no siempre son abordadas desde el ángulo neuropsicológico. Aunadas a otras comorbilidades y a diferentes niveles, el tabaquismo y la EPOC propician una serie de alteraciones fisiológicas causando, a su vez, cambios neuropsicológicos que impactan sobre la calidad de vida y el funcionamiento de los pacientes. Esta revisión resume aspectos críticos relacionados con el funcionamiento cognitivo y las enfermedades respiratorias.
\end{abstract}

Palabras clave: Adicción, EPOC, hipoxia, neuropsicología, tabaquismo.

ABSTRACT. There are several conditions and factors that may have an impact on brain functioning due to physiological changes in brain structures. In clinical practice, respiratory disease, including smoking and chronic obstructive pulmonary disease (COPD) are not always approached from a neuropsychological angle. With other comorbidities and at different levels, smoking and COPD foster a series of physiological and neuropsychological changes that have an impact on quality of life and functioning of patients. This review summarizes critical aspects of cognitive functioning and respiratory diseases.

Key words: Addiction, COPD, hypoxia, neuropsychology, smoking.

\section{EPIDEMIOLOGÍA DEL TABAQUISMO Y LA EPOC}

A nivel mundial, aproximadamente 6 millones de personas mueren al año por el consumo del tabaco, tanto por su uso directo como por humo de segunda mano. Para el 2020 se estima que esta cifra incrementará a 7.5 millones. ${ }^{1}$ Son ampliamente conocidas las enfermedades asociadas con esta adicción: cáncer de pulmón, 71\%; enfermedades respiratorias (incluyendo la EPOC), $42 \%$; y casi $10 \%$ de enfermedades cardiovasculares, colocando al tabaquismo como el problema de salud pública más importante. ${ }^{2}$

En América Latina mueren más de 400 personas cada día como consecuencia del tabaquismo. A la fecha, se estima que hay 150 mil muertes anuales en América Latina y el Caribe atribuidas al uso de este producto. ${ }^{3,4}$ Tan solo en México casi 68 de cada 100 personas de 18 a 29 años de edad consumió tabaco alguna vez en su vida, provocando problemas no sólo relacionados a la salud, sino también sociales debido a los costos del consumo, el medio ambiente y la calidad de vida del individuo fumador y quien vive con él. En México, para el año 2020 el tabaco estará ocasionando el fallecimiento de 400 mil personas cada año. ${ }^{5}$ Datos de la Organización Panamericana de la Salud (OPS) de 2005 indican que la proporción de consumo de tabaco en adultos en el país es del $8.3 \%$. Este porcentaje coloca a México por encima de países como Ecuador, Guatemala y Costa Rica con $4.8 \%, 4.1 \%$ y $3.0 \%$, respectivamente. ${ }^{1}$

La Encuesta Nacional de Salud y Nutrición 2006 señala que casi 47 de cada 100 varones de 20 años a nivel nacional, han fumado al menos 100 cigarrillos durante su vida, mientras que poco más de 15 de cada 100 mujeres comparte esa característica. Asimismo, el número de personas de 18 a 65 años que fuma diariamente representa $54.1 \%$ del total de fumadores; más de medio millón consume 20 y más cigarrillos al día. Para ambos sexos, más de $60 \%$ fuma entre uno y cinco cigarrillos diarios. ${ }^{1}$ 
Estos datos reflejan que el consumo del tabaco y la exposición a su humo se mantienen como la primera causa de muerte prevenible en el mundo, tanto de fumadores activos, como a causa del humo pasivo. El problema del consumo de tabaco no sólo reside en las muertes a las que se asocia, sino también a la disminuida calidad de vida que se observa en los fumadores debido al deterioro y las complicaciones que ocasionan las comorbilidades como: cáncer de pulmón, laringe, riñón, vejiga, estómago, colon, cavidad oral, esófago, EPOC, asma, diabetes, cardiopatía isquémica, aborto, parto prematuro, problemas del estado de ánimo como depresión mayor y ansiedad ${ }^{6,7}$ problemas cognitivos, etc. ${ }^{8}$

Una de las graves consecuencias del tabaquismo son las enfermedades respiratorias, entre ellas la EPOC que representa una fuerte disminución en la calidad de vida del paciente por la cronicidad de los síntomas y la necesidad de hospitalizaciones en caso de exacerbación. En México, aproximadamente el $70 \%$ de los casos de EPOC se asocian al tabaquismo y el restante a la exposición crónica al humo de biomasa. Esto no quiere decir que todos los fumadores desarrollan EPOC (alrededor del 15 y $20 \%$ de ellos), sino que la principal causa de la enfermedad es el tabaquismo. La prevalencia mundial de la EPOC en sujetos mayores de 45 años se estima que puede ser de 3 a $10 \%$, lo cual la coloca en el cuarto lugar de causa de muertes a nivel global, junto con el sida. En el país tiene una prevalencia alrededor del $7.8 \%{ }^{9}$ y ocupa el cuarto lugar en mujeres y el quinto en hombres dentro de las 10 primeras causas de mortalidad general. ${ }^{1,9,10}$

\section{ADICCIÓN A LA NICOTINA DEL TABACO}

El tabaquismo se define como un síndrome de daño multisistémico lentamente progresivo asociada a la adicción a la nicotina. ${ }^{11}$ Resulta un desorden crónico que se caracteriza por la pérdida de control sobre el fumar, por la aparición de síntomas de abstinencia y recaídas después de un determinado tiempo de ésta. ${ }^{3}$

La dependencia psicológica resulta de las múltiples asociaciones entre el placer generado por la nicotina, la acumulación de las experiencias vivenciales y la construcción de la estructura imaginativa que hace la persona que fuma tabaco durante su vida íntima y colectiva. $^{12}$ Ambos estados, tanto psicológicos como propiamente físicos, tienen su origen en el cerebro. Para entenderlo, existen estructuras y mecanismos neurobiológicos relacionados al consumo. ${ }^{13,14}$ Éstos a su vez, están vinculados con otros como los emocionales y de aprendizaje por asociación. A partir de ellos podemos explicar el aprendizaje entre estímulos neutrales y los efectos de la nicotina. Esto supone un procesamiento en el cual están implicadas diferentes estructuras y circuitos, partiendo principalmente del área ventral tegmental y la liberación de dopamina en áreas límbicas y corteza prefrontal. La corteza prefrontal es responsable de la asociación de información (ambiente-recompensa). Entre las principales estructuras límbicas implicadas está la amígdala, encargada de procesar el significado emocional de los estímulos presentados y disparar la respuesta psicológica y fisiológica que motiva la conducta (figura 1). ${ }^{15}$ Dicho aprendizaje también se vincula con el efecto de gratificación por el uso de drogas, siendo una de las principales razones por la cual los seres humanos las consumen. ${ }^{16,17}$

\section{TABAQUISMO Y FUNCIONAMIENTO COGNITIVO}

Existen diversas enfermedades o factores que pueden causar daño o tener un impacto, ya sea permanente $o$ reversible, sobre el funcionamiento cognitivo. En el caso de las adicciones, se han estudiado diferentes cambios cerebrales con drogas ilícitas como la cocaína ${ }^{17}$ y con drogas lícitas como es el caso del tabaco. ${ }^{8}$ En diferentes niveles estas modificaciones se han observado y establecido, tanto de manera estructural como funcional y neuroquímicamente. ${ }^{13,18} \mathrm{En}$ el caso del tabaquismo, por la naturaleza de la adicción, hasta hace algunos años existía la controversia acerca del daño ${ }^{19}$ o beneficio ${ }^{20}$ que la nicotina puede tener sobre el funcionamiento cognitivo. Por un lado, algunos de los beneficios eran argumentados o justificados a partir del impacto sobre procesos de memoria y atención mediados por vías colinérgicas ${ }^{21} \mathrm{y} / \mathrm{o}$ por el uso terapéutico a partir de la naturaleza de algunos padecimientos (como la enfermedad de Parkinson). ${ }^{22}$ Por otro lado, el daño se ha observado en estudios longitudinales ${ }^{23}$ donde este impacto, considerado como benéfico, es únicamente a corto plazo. ${ }^{20} \mathrm{~A}$ través del tiempo se ha hecho evidente que con la exposición continua al tabaco hay daño lento y progresivo en el cerebro de la misma manera que en otros órganos, desencadenando una serie de cambios y deterioro cognitivo..$^{23,24}$

\section{EL IMPACTO DE LA EPOC SOBRE EL FUNCIONAMIENTO CEREBRAL}

\section{Efectos neurocognitivos a causa de la hipoxemia crónica en la EPOC}

Hoy, la EPOC se define como una enfermedad sistémica que se presenta como una obstrucción crónica al flujo aéreo, casi siempre progresiva y parcialmente reversible. La enfermedad está asociada a una reacción 


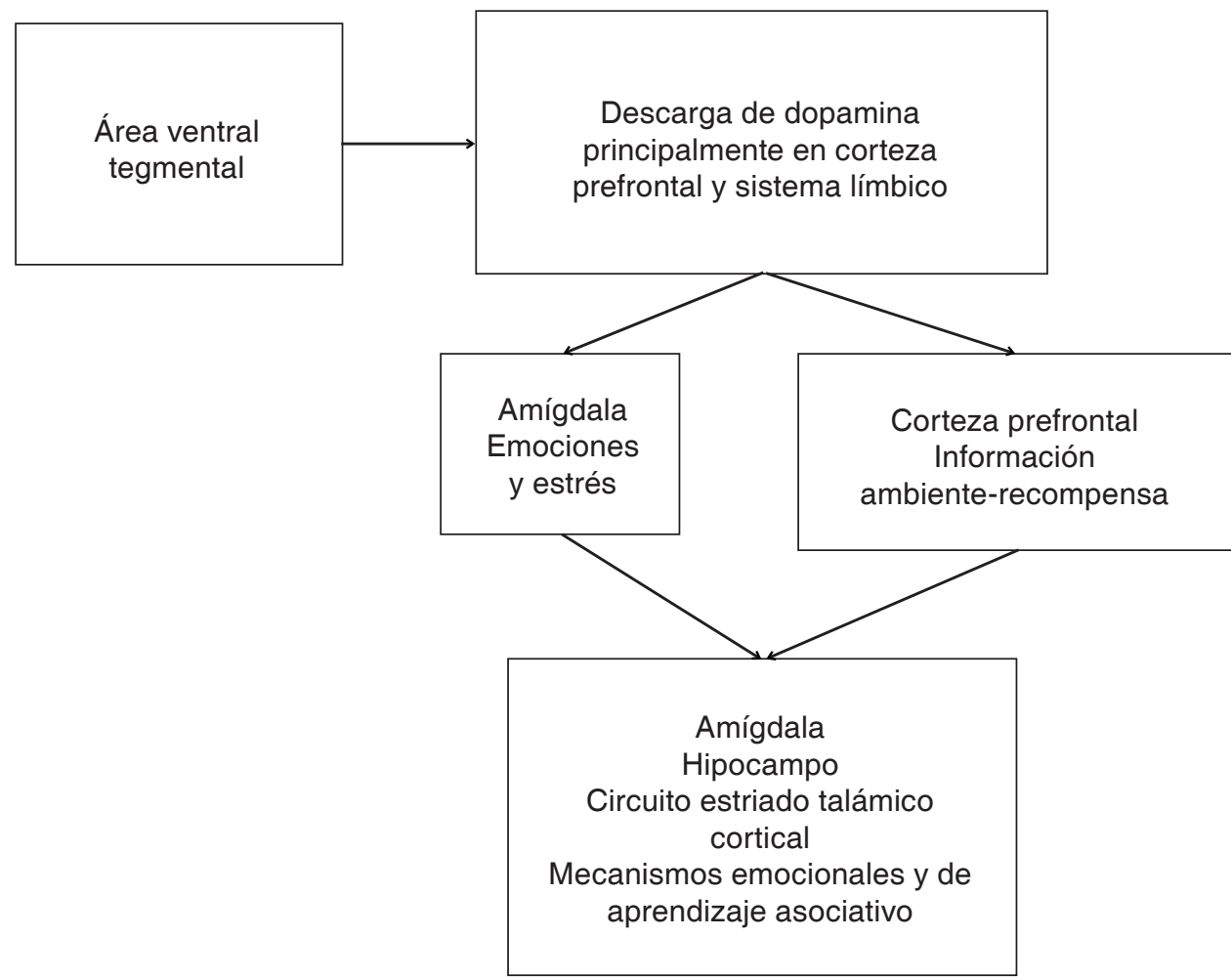

Figura 1.

Conexiones entre estructuras a partir de las cuales podemos explicar el aprendizaje entre estímulos neutrales y los efectos de la nicotina. inflamatoria pulmonar persistente ocasionada sobre todo por humo de tabaco y leña, que puede estar o no acompañada de síntomas (disnea, tos y expectoración), exacerbaciones, efectos extrapulmonares y enfermedades concomitantes. ${ }^{25}$

La disminución crónica en el flujo de aire que se presenta en el curso de la enfermedad tiene diversos efectos sistémicos; entre ellos, la desoxigenación e hipoperfusión cerebral. ${ }^{26}$ Para determinar la presencia de hipoxemia crónica es necesario realizar una gasometría, aun cuando existan signos clínicos de insuficiencia respiratoria. Los valores gasométricos varían de acuerdo con la altura de la ciudad. En la Ciudad de México los valores normales de la gasometría son: $\mathrm{PaO}_{2}$ 60-66 mmHg, pH: 7.35 a $7.45, \mathrm{PaCO}_{2}$ : $28-32 \mathrm{mmHg}_{\text {y }} \mathrm{HCO}_{2}$ : $18 \mathrm{mmHg}^{27}$

Un episodio agudo de falta de oxígeno tiene un enorme impacto en la pérdida neuronal y el deterioro cognitivo. Concentraciones de oxígeno entre 35 y 50 $\mathrm{mmHg}$ se consideran una hipoxemia moderada; por debajo de $35 \mathrm{mmHg}$ el sujeto pierde la conciencia. ${ }^{28}$ Sin embargo, al hablar de hipoxemia crónica, es difícil decir a qué nivel de $\mathrm{PaO}_{2}$ debe llegar una persona, por cuánto tiempo y con qué frecuencia deben presentarse los episodios de falta de oxígeno para que se produzca un daño neuronal que se traduzca en deterioro cognitivo medible. ${ }^{28}$ De hecho, algunas veces al indicar que el paciente necesita oxigenoterapia crónica domiciliaria no se evalúa si el paciente está en un período de hipoxemia transitoria o si realmente se trata de un problema de hipoxemia crónica que requiere esta estrategia de tratamiento. ${ }^{29}$ Además de observar los valores de la gasometría arterial, también es importante tomar en cuenta con qué frecuencia una persona presenta desaturación de oxígeno, si es en reposo o en actividad y por cuánto tiempo, para tener un panorama más claro de cuáles son las necesidades de oxígeno de la persona. ${ }^{30}$ Los estudios que han relacionado la hipoxemia crónica con el deterioro cognitivo se basan en una medida transversal de los valores de oxigenación de los pacientes ${ }^{31} \mathrm{y}$, por lo tanto, no se mide el efecto de los episodios de desaturación a lo largo del tiempo.

\section{Mecanismos fisiopatológicos de la hipoxemia}

La supervivencia de todo el organismo está asociada a cambios en los sistemas cardiovascular y respiratorio generados a partir de la detección de los quimiorreceptores de oxígeno en condiciones de hipoxia, y su «activación» procura mantener el suministro de oxígeno a los tejidos. ${ }^{32}$

Después de una disminución en la presión parcial de oxígeno $\left(\mathrm{PaO}_{2}\right)$, los quimiorreceptores tipo I de los cuerpos carotídeos liberan neurotransmisores que 
provocan la estimulación de los centros respiratorios del tallo cerebral y provocan un aumento reflejo en la ventilación. ${ }^{33}$ Las estructuras que participan en la acción de controlar las actividades del sistema nervioso simpático son el tálamo, hipotálamo, tallo cerebral, puente y médula. ${ }^{32,33}$

Si bien, la «activación» de estas neuronas quimiorreceptoras de oxígeno genera un mejoramiento de actividades respiratorias y simpáticas, ${ }^{32}$ también suceden otros cambios que pueden afectar los procesos cognitivos. Las neuronas modifican su actividad en respuesta a la hipoxia bajando su tasa metabólica, reduciendo la producción de adenosintrifosfato a través de fosforilación oxidativa. El metabolismo de la acetilcolina y de los aminoácidos neurotransmisores, la homeostasis del calcio y los niveles de catecolaminas se ven alterados, afectando a su vez la actividad neuronal. ${ }^{34}$

Esta cadena de mecanismos fisiológicos en el sistema nervioso central está relacionada a la optimización de la función respiratoria, no obstante también repercute en el rendimiento cognitivo y la conducta de una persona. Se ha descrito que las estructuras cerebrales más vulnerables al daño por hipoxemia son las que se encuentran en el extremo del suministro vascular o con mayor tasa metabólica; éstas incluyen la neocorteza, el hipocampo (principalmente la capa CA1), ${ }^{35,36}$ los ganglios basales, las células de Purkinje en el cerebelo y el tálamo. ${ }^{26}$ El patrón y la extensión de la disfunción cognitiva reportados en la EPOC varía en los pacientes y parece estar asociada con la gravedad de la enfermedad. ${ }^{37}$ En el 2001, Tae et al., realizaron un análisis de correlación entre niveles de $\mathrm{PaO}_{2}$ y actividad metabólica cerebral, encontraron que la disfunción cognitiva está asociada a los niveles de $\mathrm{PaO}_{2}$, aunque no se hallaron marcadores metabólicos característicos para pacientes con hipoxemia crónica. ${ }^{38}$

\section{INTERACCIÓN ENTRE SISTEMAS}

Existe una alta correlación entre la EPOC y otros padecimientos respiratorios con afecciones del sistema cardiovascular e inmunológico. Se ha encontrado que en las personas con afecciones respiratorias crónicas como la EPOC existe un índice importante de hipoperfusión cerebral. ${ }^{26}$ Además, se ha asociado la disfunción respiratoria con la presencia de estrés oxidativo y con disfunción vascular, lo que compromete aún más el estado cognitivo. ${ }^{39}$

Al respecto, se ha descrito que la suma de comorbilidades incrementa el riesgo de deterioro cognitivo; además, existe una relación entre el padecimiento de enfermedades respiratorias y cardiovasculares y el deterioro cognitivo leve y la demencia. ${ }^{40}$ Es interesante cuestionarse la pertinencia de relacionar estas entidades nosológicas, ya que es difícil establecer si puede diagnosticarse deterioro cognitivo leve o demencia estando comprometido el funcionamiento respiratorio y/o cardiovascular cuando los criterios diagnósticos excluyen alguna condición subyacente que pueda ser causa del deterioro, y en este caso que ocasiona daño neurofisiológico.

\section{HALLAZGOS NEUROPSICOLÓGICOS EN LA EPOC}

En los estudios que han evaluado el desempeño cognitivo de las personas con EPOC se han utilizado diversos instrumentos. Algunos han utilizado el MMSE (del inglés mini mental state examination) ${ }^{41}$ una prueba de tamizaje para detectar deterioro cognitivo. Pese a que se ocupa como «estándar de oro» en los servicios de neurología y es muy sensible a la detección de daño, es poco específico para determinar qué dominios cognitivos se encuentran afectados. ${ }^{42}$ Estudios que han utilizado otras baterías neuropsicológicas más extensas y específicas aportan mayor evidencia de las alteraciones que aqueIlos que utilizan solamente pruebas de tamizaje. Si bien, el MMSE puede detectar confiablemente la presencia de deterioro, es importante detectar las sutilezas, ya que esto puede contribuir en el manejo del paciente para mejorar su funcionalidad.

La evaluación neuropsicológica se realiza cuando se trata de establecer el estado cognitivo de un paciente. Se examina cada proceso mental para determinar la presencia de síndromes cognitivos y/o comportamentales (afasia, amnesia, demencia, etc.) y puede sugerir la etiología de la condición patológica, su topografía, su posible evolución y cuáles podrían ser las medidas de rehabilitación y manejo para el paciente. ${ }^{43}$

Al hacer una evaluación neuropsicológica se determina el desempeño cognitivo contemplando las características individuales y su contexto en factores tales como: edad, escolaridad, idioma, lugar donde fue criado, ocupaciones actuales y anteriores, factores de riesgo y antecedentes patológicos. Todo esto se suma al análisis de su desempeño en las pruebas y se obtiene un panorama de lo que una persona puede realizar sin dificultad, en qué destaca y qué habilidades ha perdido o nunca adquirió. Esto debe poder traducirse en actividades de la vida cotidiana y cómo la persona funciona en su medio. ${ }^{44}$ Además de los procedimientos puramente clínicos y cualitativos, siempre se deben emplear procedimientos estandarizados y cuantitativos de evaluación para poder transmitir la información de un profesional a otro. ${ }^{43}$

Además de la prueba MMSE, algunos de los instrumentos utilizados con mayor frecuencia en la evaluación 
de pacientes con EPOC son: el Trail Making Test, ${ }^{45-47}$ pruebas de fluidez verbal y la Escala Wechsler de Inteligencia para Adultos (WAIS), ${ }^{45,48}$ particularmente algunas subpruebas como retención de dígitos y codificación de símbolos. ${ }^{49-51}$ La prueba de Trail Making Test evalúa la capacidad que tiene una persona de búsqueda visual, velocidad de procesamiento, flexibilidad mental y funcionamiento ejecutivo (particularmente alternar secuencias), y también puede arrojar información sobre la atención dividida y la memoria de trabajo, procesos que están involucrados en la mayoría de las tareas neuropsicológicas. ${ }^{52}$ Las pruebas de fluidez verbal consisten en la evaluación de la producción verbal de una persona, implicando áreas del lenguaje (lóbulo temporal izquierdo) y áreas asociadas a la búsqueda específica de vocabulario apegándose a la fonología o categorías semánticas (lóbulo frontal). ${ }^{53}$ La WAIS fue utilizada para evaluar la inteligencia desde mediados del siglo XX. Es uno de los instrumentos más utilizados en la medición de inteligencia, entendida como un constructo que comprende habilidades verbales y no verbales. La batería permite utilizar las subpruebas para evaluar algunos procesos en particular, sin necesariamente tomar todo el conjunto de puntajes que brinda el coeficiente intelectual. ${ }^{44}$ Algunas de estas subpruebas han sido utilizadas para evaluar procesos como velocidad de procesamiento, memoria de trabajo y atención en personas con EPOC. ${ }^{45,49,50,54}$

Las alteraciones neuropsicológicas en pacientes con hipoxemia crónica no muestran un perfil específico o focalizado. ${ }^{37}$ La naturaleza de los déficits cognitivos en personas con afecciones respiratorias suele ser más bien difusa, aunque se ha detectado con frecuencia la alteración de algunos procesos en particular. ${ }^{37}$ Dado que el hipocampo es particularmente sensible a la hipoxia, uno de los procesos que con frecuencia se reportan con bajo rendimiento es la memoria. ${ }^{48,50,55-58}$ Además del hipocampo, otras estructuras que también pueden resultar afectadas por el bajo suministro de oxígeno, como la corteza de los lóbulos frontal y parietal, están involucradas en la memoria y procesos como la velocidad de procesamiento, la atención y la fluidez. ${ }^{44}$ Por lo tanto, es muy probable que si la memoria presenta deterioro (dificultad para recordar instrucciones, nombres, pendientes, etc.) otras funciones como el aprendizaje espacial, la capacidad de cambiar la atención de un estímulo a otro, las estrategias para evocar información, entre otras cosas, también se vean afectadas. ${ }^{36,59,60}$

La memoria de trabajo (una modalidad de la memoria a corto plazo) es un sistema de almacenamiento con capacidad limitada que nos permite manipular mucha información simultáneamente para desempeñar dife- rentes tareas cognitivas. ${ }^{61}$ En ello se observa un menor desempeño en pacientes con EPOC comparados con controles sanos, observándose dificultad para mantener y manipular la información, ${ }^{36,40}$ lo cual se puede relacionar directamente con bajo desempeño en tareas de fluidez verbal, tanto fonológica como semántica (capacidad para evocar palabras tanto similares en sonido como por categorías semánticas). ${ }^{45-49,54-57,62,63}$ Esto también tiene impacto en la velocidad de procesamiento, con una clara diferencia entre pacientes exacerbados, no exacerbados y personas sanas, observándose mayor lentitud en los primeros para realizar diferentes tareas. ${ }^{41,64}$

Se han reportado también alteraciones en otros dominios como habilidades visoespaciales, ${ }^{40,57,64}$ visomotoras ${ }^{45,46,54,56,62}$ y en el lenguaje. ${ }^{40,55}$ Sin embargo, en el este último dominio no es frecuente encontrar deterioro.

No existen resultados concluyentes en cuanto a una relación directa entre niveles bajos de $\mathrm{PaO}_{2}$, actividad metabólica baja en ciertas regiones cerebrales y deterioro estructural cerebral. Sin embargo, se ha observado que existe una similitud entre las características del metabolismo, el deterioro cognitivo que existe en el envejecimiento normal y el deterioro de los pacientes con EPOC. En apariencia, los cambios neurofisiológicos y neuropsicológicos en el envejecimiento normal y en la EPOC se dan de manera similar, aunque de forma acelerada en el caso de la enfermedad. Las alteraciones cognitivas se presentan de forma significativa en los pacientes con EPOC a edad más temprana, siendo más profundas en los pacientes con hipoxemia y con necesidad de utilizar oxígeno suplementario. .0,57 $^{2}$

Quienes han estudiado la posibilidad de obtener beneficios a través de la terapia de oxígeno, han encontrado que el deterioro es sólo parcialmente reversible. Queda aún mucho por indagar en cuanto a las posibilidades de intervención neurocognitiva para la rehabilitación o mantenimiento de los pacientes. ${ }^{51,57,58}$

\section{CONCLUSIÓN}

La EPOC y el tabaquismo tienen un impacto importante sobre el funcionamiento cognitivo (tabla 1), particularmente por la vulnerabilidad de algunas estructuras cerebrales a la hipoxemia, lo que desencadena una serie de eventos fisiológicos que tienen como resultado la afectación del funcionamiento cognitivo de las personas que fuman y/o con EPOC principalmente en memoria (a corto plazo), atención, habilidades visomotrices y funciones ejecutivas, particularmente en flexibilidad cognitiva. 
Tabla 1. Resume información disponible sobre la naturaleza del deterioro cognitivo por tabaquismo y EPOC, con gran utilidad para propuestas de tratamiento de rehabilitación/mantenimiento cognitivo.

\begin{tabular}{|c|c|c|c|}
\hline Autor y año & Muestra & $\begin{array}{c}\text { Test cognitivos y/o procesos } \\
\text { evaluados }\end{array}$ & Principal hallazgo/conclusión \\
\hline $\begin{array}{l}\text { Sakurai Y, et al. }{ }^{65} \\
2002\end{array}$ & $\begin{array}{l}-20 \text { fumadores de entre } 23 \text { y } \\
41 \text { años de edad con prome- } \\
\text { dio de } 14.6 \text { años fumando y } \\
22.6 \text { paquetes año } \\
\text { - } 20 \text { no fumadores }\end{array}$ & $\begin{array}{l}\text { Memoria, cálculo y asociación } \\
\text { (fluidez verbal) }\end{array}$ & $\begin{array}{l}\text { La dosis diaria de tabaco } \\
\text { impacta en memoria a corto } \\
\text { plazo, cálculo y asociación } \\
\text { mental }\end{array}$ \\
\hline $\begin{array}{l}\text { Paul RH, et al. } \\
2006\end{array}$ & $\begin{array}{l}\cdot 62 \text { fumadores ( } n=45<45 \\
\text { años de edad y } n=17>45 \\
\text { años de edad) } \\
\text { - } 62 \text { no fumadores }(n=43< \\
45 \text { años de edad y } n=19>45 \\
\text { años de edad) }\end{array}$ & $\begin{array}{l}\text { Atención, tiempos de reacción, } \\
\text { flexibilidad cognitiva, velocidad } \\
\text { motora y memoria }\end{array}$ & $\begin{array}{l}\text { Menor desempeño en tareas de } \\
\text { funcionamiento ejecutivo entre } \\
\text { fumadores y no fumadores; } \\
\text { con diferencias entre grupos } \\
\text { de edad, desempeñándose por } \\
\text { debajo los fumadores jóve- } \\
\text { nes en comparación a los no } \\
\text { fumadores }\end{array}$ \\
\hline $\begin{array}{l}\text { Yakir A, et al. }{ }^{66} \\
2007\end{array}$ & $\begin{array}{l}\text { - } 91 \text { fumadores } \\
\text { - } 40 \text { exfumadores } \\
\text { - } 151 \text { no fumadores } \\
\text { - } 46 \text { fumadores ocasionales } \\
\text { Edad: entre } 20 \text { y } 30 \text { años }\end{array}$ & $\begin{array}{l}\text { Atención, memoria, impulsivi- } \\
\text { dad, planeación, procesamien- } \\
\text { to de información y velocidad } \\
\text { motora }\end{array}$ & $\begin{array}{l}\text { Dificultades atencionales y de } \\
\text { control de impulsos entre fuma- } \\
\text { dores y no fumadores (proce- } \\
\text { sos que pueden ser factores de } \\
\text { inicio de la adicción) }\end{array}$ \\
\hline $\begin{array}{l}\text { Dodd JW, et al. }{ }^{67} \\
2013\end{array}$ & $\begin{array}{l}80 \text { pacientes con EPOC y } 30 \\
\text { controles } \\
30 \text { pacientes con reciente } \\
\text { exacerbación, } 50 \text { pacientes } \\
\text { estables }\end{array}$ & $\begin{array}{l}\text { - Memoria episódica } \\
\text { - Funcionamiento ejecutivo } \\
\text { - Habilidades visoespaciales } \\
\text { - Memoria de trabajo } \\
\text { - Velocidad de procesamiento }\end{array}$ & $\begin{array}{l}\text { Pacientes con EPOC hospi- } \\
\text { talizados con exacerbación } \\
\text { puntúan por debajo de la norma } \\
\text { en el Trail Making Test y en } \\
\text { pruebas de habilidades visoes- } \\
\text { paciales } \\
\text { Pacientes con EPOC estables } \\
\text { puntúan dentro de lo normal. } \\
\text { Algunos presentaron dificulta- } \\
\text { des de leves a moderadas en } \\
\text { tareas de memoria visual y de } \\
\text { habilidades visoespaciales }\end{array}$ \\
\hline $\begin{array}{l}\text { Li J, et al. }{ }^{68} \\
2013\end{array}$ & $\begin{array}{l}27 \text { pacientes con EPOC } \\
\text { moderado } \\
\cdot 35 \text { pacientes con EPOC grave } \\
\text { - } 27 \text { controles }\end{array}$ & MMSE & $\begin{array}{l}\text { Puntajes generales de pa- } \\
\text { cientes con EPOC en MMSE } \\
\text { menores a lo esperado en- } \\
\text { contrándose en un rango de } \\
\text { daño leve a moderado, siendo } \\
\text { proporcional con la severidad } \\
\text { de la enfermedad }\end{array}$ \\
\hline $\begin{array}{l}\text { Chang SS, et al. }{ }^{69} \\
2012\end{array}$ & $\begin{array}{l}393 \text { participantes de } \geq 65 \text { de } \\
\text { edad }\end{array}$ & Versión modificada del MMSE & $\begin{array}{l}\text { El } 65 \% \text { de los participantes del } \\
\text { estudio con problemas cogniti- } \\
\text { vos fueron hombres }\end{array}$ \\
\hline $\begin{array}{l}\text { Dodd JW, et al. }{ }^{57} \\
2012\end{array}$ & $\begin{array}{l}\text { - } 25 \text { pacientes con EPOC } \\
\text { - } 25 \text { controles }\end{array}$ & rfMRI & $\begin{array}{l}\text { Pacientes con EPOC no } \\
\text { hipogénicos presentan dismi- } \\
\text { nución en materia blanca, así } \\
\text { como baja activación funcional } \\
\text { en materia gris (impactando a } \\
\text { disfunción cognitiva) }\end{array}$ \\
\hline
\end{tabular}




\section{Continúa tabla 1.}

\begin{tabular}{|c|c|c|}
\hline $\begin{array}{l}\text { Pereira ED, et al. }{ }^{70} \\
2011\end{array}$ & $\begin{array}{l}34 \text { pacientes con EPOC mayo- } \\
\text { res de } 45 \text { años }\end{array}$ & $\begin{array}{l}\text { - The Stroop test } \\
\text { - The FAS (fluidez verbal) } \\
\text { - The Digit Span test } \\
\text { - The Rey Auditory Verbal Lear- } \\
\text { ning Test (RAVLT) }\end{array}$ \\
\hline
\end{tabular}
Mejorías post intervención cognitiva en aprendizaje verbal, memoria, organización y proce- samiento verbal
Se ajustó la variable nivel sociodemográfico, que podría interferir de manera importante sobre la cognición
El género masculino y la edad menor a 65 años se asociaron a mayor puntaje en aprendizaje verbal y memoria en pre y post intervención

Etnier J, et al. ${ }^{63}$ 1999

98 pacientes de 56 a 80 años

121 pacientes de en promedio 61 años de edad
- Inteligencia

- Tiempos de reacción

- Memoria de trabajo

Batería neuropsicológica Halstead-Rietan
En la población de mayor edad, la edad, el ejercicio y la función pulmonar son predictores de desempeño cognitivo. La edad y ejercicio predicen velocidad de procesamiento, misma que influye en el desempeño de las demás tareas

La mayoría de los pacientes con EPOC presentan dificultades cognitivas en abstracción y habilidades de integración motora. Las habilidades motoras simples y atencionales fueron menos prevalentes de daño. Lenguaje y memoria se encontraron intactos

Abreviaturas: $\mathrm{EPOC}=$ Enfermedad pulmonar obstructiva crónica; MMSE = Mini Mental State Examination.

\section{REFERENCIAS}

1. Instituto Nacional de Estadística, Geografía e Informática. A propósito del día del tabaco. México: INEGI, 2008. Fecha de consulta: julio, 2014. Disponible en: www.inegi.org.mx/inegi/contenidos/espanol/prensa/.../ tabaco05.pdf.

2. World Health Organization. Tabaquismo. 2011. Fecha de consulta: julio, 2014. Disponible en: http://www.who. int/topics/tobacco/es/

3. Sansores MR, Ramírez-Venegas A. Enfermedad pulmonar obstructiva crónica y la celebración de su primer Día Mundial. Rev Inst Nal Enf Resp Mex 2002; 15(4):199-200.

4. Sansores R, Ramírez-Venegas A, Espinosa M, Sandoval R. Tratamientos para dejar de fumar, disponibles en México. Salud Publica Mex 2002;44 supl 1):S116-S124. http://dx.doi.org/10.1590/S0036-36342002000700017.

5. Secretaría de Salud. Programa de Acción. Adiciones. Tabaquismo. México, 2001. Fecha de consulta: julio, 2014. Disponible en: http://www.salud.gob.mx/unidades/cdi/ documentos/tabaquismo.pdf.
6. Murray CJ, Lopez AD. Measuring the global burden of disease. N Engl J Med 2013;369(5):448-457.doi:10.1056/ NEJMra1201534.

7. Paul RH, Brickman AM, Cohen RA, et al. Cognitive status of young and older cigarette smokers: data from the international brain database. J Clin Neurosci 2006;13(4):457-465.

8. Jasinska AJ, Zorick T, Brody AL, Stein EA. Dual role of nicotine in addiction and cognition: a review of neuroimaging studies in humans. Neuropharmacology 2014;84:111-122. doi:10.1016/j.neuropharm.2013.02.015.

9. Sansores RH, Ramírez-Venegas A, Hernández-Zenteno R, Mayar-Maya ME, Pérez-Bautista OG, Velázquez Uncal M. Prevalence and diagnosis of chronic obstructive pulmonary disease among smokers at risk. A comparative study of case-finding vs. screening strategies. Respir Med 2013;107(4):580-586. doi: 10.1016/j.rmed.2012.12.010.

10. Guías para el Diagnóstico y Tratamiento de la Enfermedad Pulmonar Obstructiva Crónica. Derivadas del Cuarto Consenso Mexicano para el Diagnóstico y Tratamiento de la EPOC. Neumol Cir Torax 2012;71(1 supl):S8-S89. 
11. Benowitz NL. Nicotine addiction. N Engl J Med 2010;362(24):2295-2303. doi: 10.1056/NEJMra0809890.

12. American Psychiatric Association. DSM-IV-TR. Manual diagnóstico y estadístico de los trastornos mentales. Barcelona: Masson; 2002.p.181-199.

13. Volkow ND, Baler RD. Addiction science: Uncovering neurobiological complexity. Neuropharmacology 2014;76 Pt B:235-249. doi: 10.1016/j.neuropharm.2013.05.007

14. Baler RD, Volkow ND. Drug addiction: the neurobiology of disrupted self-control. Trends Mol Med 2006;12(12):559566. doi:10.1016/j.molmed.2006.10.005.

15. Muñoz MÁ, Sanjuan R, Fernández-Santaella MC, Vila J, Montoya P. Neuropsychological aspects of nicotine craving. Adicciones 2011;23(2):111-123.

16. Volkow ND, Wang GJ, Fowler JS, Tomasi D. Addiction circuitry in the human brain. Annu Rev Pharmacol Toxicol 2012;52:321-336. doi:10.1146/annurevpharmtox-010611-134625.

17. Spronk DB, van Wel JH, Ramaekersc JG, Verkes RJ. Characterizing the cognitive effects of cocaine: a comprehensive review. Neurosci Biobehav Rev 2013;37(8):18381859. doi: 10.1016/j.neubiorev.2013.07.003.

18. Zhang X, Salmeron BJ, Ross TJ, et al. Anatomical differences and network characteristics underlying smoking cue reactivity. Neuroimage 2011;54(1):131-141. doi: 10.1016/j.neuroimage.2010.07.063.

19. Durazzo TC, Meyerthoff DJ, Nixon SJ. Chronic cigarette smoking: implications for neurocognition and brain neurobiology. Int J Environ Res Public Health 2010;7(10):3760-3791. doi: 10.3390/ijerph7103760.

20. Rusted JM, Sawyer R, Jones C, Trawley SL, Marchant NL. Positive effects of nicotine on cognition: the deployment of attention for prospective memory. Psychopharmacology (Berl) 2009;202(1-3):93-102.doi:10.1007/ s00213-008-1320-7.

21. Mansvelder HD, van Aerde KI, Couey JJ, Brussaard A. Nicotinic modulation of neuronal networks: from receptors to cognition. Psychopharmacology (Berl) 2006;184(3-4):292-305.

22. Ríčný J. Overlooked Alzheimer's smoking gun? Neurochem Res 2013;38(9): 1774-1776. doi10.1007/s11064-013-1086-0.

23. Almeida OP, Garrido GJ, Alfonso $\mathrm{H}$, et al. 24-month effect of smoking cessation on cognitive function and brain structure in later life. Neuroimage 2011;55(4):14801489. doi: 10.1016/j.neuroimage.2011.01.063.

24. Fried PA, Watkinson B, Gray R. Neurocognitive consequences of cigarette smoking in young adults--a comparison with pre-drug performance. Neurotoxicol Teratol 2006;28(4):517-525.

25. Iniciativa Global para la Enfermedad Pulmonar Obstructiva Crónica. Guía para el Diagnóstico, Tratamiento y Prevención de la EPOC. Una Guía para profesionales sanitarios. EUA, 2001.p.6-10. Fecha de consulta: julio, 2014. Disponible en: http://www.fisiorespiracion.es/gold.pdf

26. Mendoza JE, Foundas AL. The vascular system. In: Clinical neuroanatomy: A neurobehavioral approach. New York: Springer;2008.p.520-523.

27. Consenso Mexicano de EPOC. Intervenciones prioritarias. Neumol Cir Torax 2007;66(sup 2):S26-S31.
28. Areza-Fegyveres R, Kairalla RA, Carvalho CR, Nitrini R. Cognition and chronic hypoxia in pulmonary diseases. Dement Neuropsychol 2010;4(1):14-22.

29. Torre-Bouscoulet T, Vázquez-García JC, Pérez-Padilla R. Oxigenoterapia crónica domiciliaria en altitudes moderadas. Un enorme reto en salud. Neumol Cir Torax 2011;70(3):149-151.

30. Vargas-Domínguez C, Gochicoa-Rangel L, VelázquezUncal M, et al. Pruebas de función respiratoria, ¿cuál y a quién? Neumol Cir Torax 2011;70(2):101-117.

31. Dodd JW, Getov SV, Jones PW. Cognitive function in COPD. Eur Respir J 2010;35(4):913-922. doi: 10.1183/09031936.00125109.

32. Kuwaki, T. Hypothalamic modulation of breathing. Adv Exp Med Biol 2010;669:243-247. doi: 10.1007/978-14419-5692-7_49.

33. Patel AJ, Honoré E. Molecular physiology of oxygen-sensitive potassium channels. Eur Respir J 2001;18(1):221-227.

34. Virués-Ortega J, Garrido E, Javierre C, Rivero PD. Funcionamiento neuropsicológico bajo condiciones de hipoxia aguda y crónica asociada a la altitud. Arch Biol Andina 2008;14(1):40-50.

35. Kawasaki K, Traynelis SF, Dingledine R. Different responses of CA1 and CA3 regions to hypoxia in rat hippocampal slice. Neurophysiol 1990;63(3):385-394.

36. Di Paola M, Caltagirone C, Fadda L, Sabatini U, Serra L, Carlesimo GA. Hippocampal atrophy is the critical brain change in patients with hypoxic amnesia. Hippocampus 2008;18(7):719-728. doi: 10.1002/hipo.20432.

37. Hopkins RO, Bigler, ED. Hypoxic and anoxic conditions of the CNS. In: Morgan J, Ricker J, editors. Textbook of clinical neuropsychology. New York, EUA: Taylor \& Francis; 2008.p.521-535.

38. Shim TS, Lee JH, Kim SY, et al. Cerebral metabolic abnormalities in COPD patients detected by localized proton magnetic resonance spectroscopy. Chest 2001;120(5):1506-1513.

39. Morgan B. Vascular consequences of intermittent hypoxia. In: Roach RC, Wagner PD, Hackett PH, editors. Hypoxia and the circulation. Advances in experimental medicine and biology. New York, EUA: Springer; 2007.p.51-67.

40. Singh B, Parsaik AK, Mielke MM, et al. Chronic obstructive pulmonary disease and association with mild cognitive impairment: the Mayo Clinic Study of Aging. Mayo Clinic Pro 2013;88(11):1222-1230. doi: 10.1016/j.mayocp.2013.08.012.

41. Tulek B, Atalay NB, Yildirim G, Kanat F, Süerdem M. Cognitive function in chronic obstructive pulmonary disease: relationship to global initiative for chronic obstructive lung disease 2011 categories. Respirology 2014;19(6):873-880.doi: 10.1111/resp.12333.

42. Folstein MF, Folstein SE, McHugh PR. "Mini-mental state". A practical method for grading the cognitive state of patients for the clinician. J Psychiatr Res 1975;12(3):189198.

43. Ardila A, Ostrosky F. Guía para el diagnóstico neuropsicológico. 2012,127-152. Fecha de consulta: julio, 2014. Disponible en: http://www.ineuro.cucba.udg.mx/libros/ bv_guia_para_el_diagnostico_neuropsicologico.pdf. 
44. Lezak M. Neuropsychological assessment. 4th ed. New York: Oxford University Press; 2004.p.714.

45. Kozora E, Emery CF, Ellison MC, Wamboldt FS, Díaz PT, Make P. Improved neurobehavioral functioning in emphysema patients following lung volume reduction surgery compared with medical therapy. Chest 2005;128(4):2653-2663.

46. Kozora E, Emery CF, Zhang L, Hoth KF, Murphy J, Make B; National Emphysema Treatment Trial Research Group. Sequential cognitive skills in emphysema patients foIlowing lung volume reduction surgery: a 2-year longitudinal study. J Cardiopulm Rehabil Prev 2011;31(6):386391. doi: 10.1097/HCR.0b013e3182343bc8.

47. Dal Negro RW, Bonadiman L, Tognella S, Bricolo FP, Turco P. Extent and prevalence of cognitive dysfunction in chronic obstructive pulmonary disease, chronic nonobstructive bronchitis, and in asymptomatic smokers, compared to normal reference values. Int J Chron Obstruct Pulmon Dis 2014;9:675-683. doi: 10.2147/COPD. S63485.

48. Ortapamuk H, Naldoken S. Brain perfusion abnormalities in chronic obstructive pulmonary disease: comparison with cognitive impairment. Ann Nucl Med 2006;20(2):99-106.

49. Crews WD, Jefferson AL, Bolduc T, et al. Neuropsychological dysfunction in patients suffering from end-stage chronic obstructive pulmonary disease. Arch Clin Neuropsychol 2001;16(7):643-652.

50. Borson S, Scanlan J, Friedman S, et al. Modeling the impact of COPD on the brain. Int J Chron Obstruct Pulmon Dis 2008;3(3):429-434.

51. Kent BD, Mitchell PD, McNicholas WT. Hypoxemia in patients with COPD: causes, effects, and disease progression. Int J Chron Obstruct Pulmon Dis 2011;6:199. 208. doi: 10.2147/COPD.S10611.

52. Tombaugh TN. Trail Making Test $A$ and B: Normative data stratified by age and education. Arch Clin Neuropsychol 2004;19(2):203-214.

53. Tombaugh TN, Kazak J, Rees L. Normative data stratified by age and education for two measures of verbal fluency: FAS and animal naming. Arch Clin Neuropsychol 1999;14(2):167-177.

54. Liesker JJ, Postma DS, Beukema RJ, et al. Cognitive performance in patients with COPD. Respir Med 2004;98(4):351-356.

55. De Carolis A, Giubilei F, Caselli G, et al. Chronic obstructive pulmonary disease is associated with altered neuropsychological performance in young adults. Dement Geriatr Cogn Dis Extra 2011;1(1):402-408. doi: 10.1159/000333079.

56. Grant I, Heaton RK, McSweeney AJ, Adams KM, Timms RM. Brain dysfunction in COPD. Chest 1980;77(2 Suppl):S308-S309.

57. Dodd JW, Chung AW, van den Broek MD, Barrick TR, Charlton RA, Jones PW. Brain structure and function in chronic obstructive pulmonary disease: a multimodal cranial magnetic resonance imaging study. Am J Respir Crit Care Med 2012;186(3):240-245. doi:10.1164/ rccm.201202-03550C.
58. Kaptein AA, Scharloo M, Fischer MJ, et al. 50 years of psychological research on patients with COPD-road to ruin or highway to heaven? Respir Med 2009;103(1):311. doi: 10.1016/j.rmed.2008.08.019.

59. Warrington JP, Ashpole N, Csiszar A, Lee YW, Ungvari $\mathrm{Z}$, Sonntag WE. Whole brain radiation-induced vascular cognitive impairment: mechanisms and implications. J Vasc Res 2013;50(6):445-457.doi:10.1159/000354227.

60. Schega L, Peter B, Törpel A, Mutschler H, Isermann B, Hamacher D. Effects of intermittent hypoxia on cognitive performance and quality of life in elderly adults: a pilot study. Gerontology 2013;59(4):316-323.doi:10.1159/000350927.

61. Portellano JA. Introducción a la neuropsicología. Madrid: McGrawHill; 2005.p.233-239.

62. Emery CF, Green MR, Suh S. Neuropsychiatric function in chronic lung disease: the role of pulmonary rehabilitation. Respir Care 2008;53(9):1208-1216.

63. Etnier J, Johnston R, Dagenbach D, Pollard RJ, Rejeski WJ, Berry M. The relationship among pulmonary function, aerobic fitness, and cognitive functioning in older COPD. Chest 1999;116(4):953-960.

64. Antonelli-Incalzi R, Corsonello A, Pedone C. Drawing impairment predicts mortality in severe COPD. Chest 2006;130(6):1687-1694.

65. Sakurai Y, Kanazawa I. Acute effects of cigarettes in nondeprived smokers on memory, calculation and executive functions. Hum Psychopharmacol 2002; 17(7):369-373. doi:10.1002/hup.424.

66. Yakir A, Rigbi A, Kanyas K, et al. Why do young women smoke? III. Attention and impulsivity as neurocognitive predisposing factors. Eur Neuropsychopharmacol 2007;17(5):339-351.

67. Dodd JW, Charlton RA, van den Broek MD, Jones PW. Cognitive dysfunction in patients hospitalized with acute exacerbation of COPD. Chest 2013;144(1):119-127. doi:10.1378/chest.12-2099.

68. Li J, Huang Y, Fei GH. The evaluation of cognitive impairment and relevant factors in patients with chronic obstructive pulmonary disease. Respiration 2013;85(2):98105.doi:10.1159/000342970.

69. Chang SS, Chen S, McAvay GJ, Tinetti ME. Effect of coexisting chronic obstructive pulmonary disease and cognitive impairment on health outcomes in older adults. J Am Geriatr Soc 2012;60(10):1839-1846.doi:10 $.1111 / \mathrm{j} .1532-5415.2012 .04171$.

70. Pereira ED, Viana CS, Taunay TC, Sales PU, Lima JW, Holanda MA. Improvement of cognitive function after a three-month pulmonary rehabilitation program for COPD patients. Lung 2011;189(4):279-285.doi:10.1007/s00408-011-9303-6.

\section{$\triangle$ Correspondencia:}

Mtra. Valeri Noé Díaz, Instituto Nacional de Enfermedades Respiratorias Ismael Cosío Villegas. Calzada de Tlalpan Núm. 4502, Colonia Sección XVI, México, D.F., 14080.

Teléfono 54871742

Correo electrónico: valerinp@ hotmail.com

Los autores declaran no tener conflicto de intereses 